\title{
Atenção à saúde na síndrome demencial: qual será o impacto econômico dessa atenção no Brasil?
}

\author{
Health care in dementia: what will be the economic impact of this \\ care in Brazil?
}

Leyla Gomes Sancho ${ }^{1}$

\begin{abstract}
RESUMO O estudo pretende chamar a atenção dos tomadores de decisão para a premente necessidade da avaliação do impacto econômico da síndrome demencial no Brasil. O País já se enquadra, em termos demográficos e epidemiológicos, em patamar semelhante ao dos países desenvolvidos, mas ainda não implementou de forma efetiva políticas públicas ou reconheceu as reais necessidades sociais em saúde para a configuração dessa atual condição. Para tanto, uma revisão não sistemática sobre o impacto financeiro da síndrome em várias regiões do mundo foi elaborada. Diante das evidências, concluiu-se que é de suma importância a realização de estudos empíricos no contexto brasileiro que contemplem essa temática.
\end{abstract}

PALAVRAS-CHAVE Demência; Alocação de custos; Governo; Políticas públicas.

\begin{abstract}
The study intends to draw the attention of decision makers for the urgent need to assessment the economic impact of dementia in Brazil. Nowadays, this country stands at the same baseline, in demographic and epidemiological terms, as developed countries. However, it has not yet implemented policies or even recognized real health needs to this current condition. Therefore, a non-systematic review of the financial impact of the syndrome in various regions of the world was developed. Given the evidence, it was concluded that it is extremely important to carry out empirical studies in the Brazilian context that address this theme.
\end{abstract}

KEYWORDS Dementia; Cost allocation; Government; Public policies. 


\section{Introdução}

Como amplamente aludido no meio acadêmico, a síndrome demencial já se tornou um problema de saúde pública (VERAS ET AL., 2007). Sua ocorrência é crescente, uma vez que o principal fator de risco continuará existindo: o aumento da esperança de vida. Ademais, e como consequência da incessante inovação tecnológica, novas causas são esclarecidas ou descobertas, apesar das dificuldades e disparidades no seu diagnóstico. Existe um consenso em reconhecer que a maior parte das síndromes demenciais é devida à Doença de Alzheimer (MORAGAS, 1994), o que foi corroborado por recente estudo de Gallucci, Tamelini e Forlenza (2005), ao afirmar que $60 \%$ dos casos têm essa doença como causa, seguida pelas demências vasculares, pela demência por Corpúsculos de Lewy e pela demência frontotemporal.

De acordo com o 'World Alzheimer Report 2010', cerca de 35,6 milhões de cidadãos no mundo convivem com algum tipo de síndrome demencial, sendo que dois terços destes moram em países de baixa e média renda, aí incluído o Brasil. Além disso, ainda segundo o relatório, é previsto que em 2050 o quantitativo de pessoas acometidas pela síndrome chegue a 115,4 milhões (WIMO; PRINCE, 2010).

No Brasil, e independentemente do fato de se inscreverem os idosos como 'bombas demográficas', poucos foram os estudos de base populacional realizados para medir a prevalência da síndrome demencial (ALMEIDA; SANTANA; PINHO, 1984; BLAY; MARI; RAMOS, 1989; VERAS; COUTINHO, 1991; HERRERA ET AL., 2002). Tomando o de Herrera et al. (2002), que avaliou a prevalência da doença e sua associação com idade, sexo, nível educacional e níveis socioeconômicos, a estimativa referida foi de $7,1 \%$ entre idosos não institucionalizados e de 7,5\% no cômputo geral, incluindo os institucionalizados. As associações, medidas através de análise de regressão multivariada, mostraram que o baixo nível educacional, o sexo feminino e a idade são estatisticamente significativos.
Esses resultados são de especial interesse para esta discussão, em vista de favorecerem o reconhecimento das reais necessidades em relação aos cuidados em saúde e seu impacto financeiro.

Quanto ao Brasil, estudo publicado no ano de 2004 estima a incidência da síndrome em 13,8 por 1000 pessoas-ano (NITRINI ET AL., 2004). Relacionando-se ambas as informações (incidência e prevalência), esse dado não só desvela como corrobora a importância da proposição de políticas públicas de saúde capazes, se não de minimizar o impacto econômico para a sociedade, pelo menos de garantir o aporte necessário de recursos públicos para garantir a atenção ao paciente. Observa-se que, no contexto brasileiro, o orçamento destinado para suprir as demandas dos idosos ainda é muito incipiente, apesar da criação da Política Nacional de Saúde do Idoso (BRASIL, 1999), da proposição do Pacto pela Vida pelo Ministério da Saúde, que tem a saúde do idoso como uma de suas prioridades (BRASIL, 2006), e mesmo o Programa de Assistência aos Portadores de Doença de Alzheimer, estabelecido pela Portaria GM/ MS n ${ }^{0} 703 / 2002$ (BRASIL, 2002), e o Protocolo Clínico e Diretrizes Terapêuticas - Demência por Doença de Alzheimer (Portaria SAS/MS $n^{\circ} 843 / 2002$ ), que institui o fornecimento da medicação anticolinesterásica (BRASIL, 2002).

No âmbito mundial, o estudo realizado por Wimo e Prince (2010) já indicava que o custo direto da atenção aos pacientes portadores de demência seria de US\$ 640 bilhões e que $70 \%$ deste custo estaria circunscrito aos Estados Unidos e países da Europa Ocidental. Ainda na perspectiva mundial, o custo da atenção informal (relativo ao cuidador) seria de US\$ 251 bilhões e o custo direto (médico) e o não médico (também denominado na literatura internacional de custo social) da atenção formal (paciente) seria de US\$ 352 bilhões. O estudo também chama a atenção para o crescimento dos custos - cerca de $34 \%$ - entre os anos de 2005 a 2009. 
No que se refere à América Latina, outro estudo publicado em 2006, estimou a aplicação de US\$ 7,8 bilhões em custos diretos e US\$ 6 bilhões com a atenção informal, lembrando que nos países em desenvolvimento os custos não médicos (sociais) são pouco expressivos, diante da inexpressividade das políticas públicas sociais (WIMO; JONSSON; WINBLAD, 2006). Na perspectiva da discussão sobre a abrangência das políticas e na especificidade das doenças que acometem os idosos, encontrou-se na literatura que é característico o cuidado ser prestado por um sistema de suporte informal, principalmente nos países em desenvolvimento e nos emergentes. Estudos empíricos referem que o cuidado informal é parte da abordagem atual da atenção prestada, principalmente, pela família/comunidade ao paciente; observa-se, inclusive, que, em um número importante de países, e independentemente do fato de o País oferecer um sistema de saúde abrangente, esse cuidado - e seu custo subjacente - é predominante em relação ao do cuidado formal (CHAPPELL, 1993; WIMO ET AL., 2010; JONSSON; WIMO, 2009). Deve-se entender que cuidador é a pessoa, membro ou não da família, que, com ou sem remuneração, cuida do idoso doente ou dependente no exercício de suas atividades diárias.

Considerando o panorama esboçado e a premissa de que o prolongamento da vida, a dignidade e a cidadania dos idosos, assim como dos portadores e cuidadores daqueles que sofrem de síndrome demencial, é decorrência e não causa de elevação dos gastos públicos, o conhecimento acerca desses gastos mostra-se de fundamental importância para o delineamento de políticas públicas de saúde envolvendo essa condição. Por sua vez, o conhecimento dos gastos exige instrumentais adequados, a fim de contemplar as diferentes variáveis que os compõem.

Entre os questionários disponíveis para essa finalidade, o Resource Utilization in Dementia (RUD) (WIMO ET AL., 1998) mostra-se particularmente promissor, em vista de possibilitar amplo leque de leituras dos gastos e custos, tanto formais (paciente) quanto informais (cuidador).

O objetivo do presente artigo é apresentar estudos que se utilizaram do $R U D$, indicando as suas potencialidades para o planejamento e proposição de políticas públicas para a síndrome demencial.

\section{A metodologia do estudo}

O estudo realizado decorre de uma revisão não sistemática de pesquisas elaboradas $\mathrm{e}$ para as quais foi utilizado o questionário $R U D$, não apenas em países, mas também em regiões, comunidades urbanas e rurais e outros tipos de conglomerados geográficos, assim como a composição dos gastos ou custos, tanto os formais (médico e não médico do paciente) quanto os informais (relativos ao cuidador).

Para a busca desses estudos, utilizaram-se os descritores 'dementia', 'cost', 'questionnai$r e$ e 'instrument' e o termo chave 'resource utilization in dementia' nas seguintes bases de dados: PubMed, Scopus e Bireme; foram considerados elegíveis aqueles publicados em português, inglês, francês ou espanhol. Quanto à periodicidade temporal, o filtro utilizado abrangeu os estudos publicados entre os anos de 2008 a 2012, no sentido de que os resultados financeiros não apresentassem grandes distorções quanto ao poder de paridade.

É importante citar que os descritores foram dispostos na busca de forma que o termo chave (o nome do questionário) estivesse sempre presente, ou seja, utilizou-se o operador boleano 'and' para a busca.

Para processar os resumos elegíveis, definiram-se critérios de inclusão e de exclusão. Os critérios adotados para inclusão do estudo/pesquisa foram: a utilização de qualquer versão do questionário RUD (o questionário apresenta duas versões, $R U D$ e $R U D$ LITE, já traduzidas pelo grupo de pesquisa 
em vários idiomas, inclusive o português) e a apresentação como um dos resultados de valores financeiros relativos à utilização de recursos, independentemente de estarem relacionados com a atenção formal ou a informal, com o custo ou gasto total, bem como da situação de moradia do paciente. Foram também incluídos estudos que discutiram outras intervenções sociais junto aos pacientes demenciados ou cuidadores e ainda os relacionados à carga da doença dos cuidadores informais. Não houve nenhum tipo de restrição quanto aos métodos de estimação econômica praticadas para a obtenção do resultado.

Os critérios de exclusão referem-se a estudos de cunho teórico, aqueles que não demonstraram dados empíricos sobre o impacto financeiro, aqueles relativos à avaliação econômica em relação à terapia medicamentosa ou aqueles que não utilizaram o questionário RUD.

A etapa seguinte se consubstanciou na escolha das características de interesse que deveriam estar inscritas no quadro demonstrativo, e ao mesmo tempo explicativo, no que concerne aos objetivos do estudo, qual seja, o de demonstrar o impacto financeiro da síndrome demencial tanto na perspectiva da atenção formal quanto na da informal, assim como sob os pontos de vista da família, da comunidade, do governo e da sociedade.

\section{O questionário}

Em geral, o objetivo dos estudos que utilizam o RUD/RUD LITE é avaliar o incremento ou não do custo durante o período de um ano, tanto na perspectiva da evolução do grau de severidade da patologia no portador de síndrome demencial quanto no seu correspondente em relação à sobrecarga do cuidador, como também subsidiar metodologias que tenham como propósito a avaliação do impacto financeiro. A proposta é a aplicação do questionário em três etapas distintas (baseline, no sexto mês e no décimo segundo mês).

O questionário originalmente desenhado pelos pesquisadores do Instituto Karolinska (Suécia) se atém a uma avaliação dos custos, a partir do fator tempo dispendido pelos cuidadores aos portadores de síndrome demencial e o equivalente em termos de ganhos ou perdas salariais, como também o consumo de recursos de cuidados médicos e não médicos pelos pacientes e cuidadores (sobrecarga); ou seja, direcionado para avaliar tanto os custos relacionados à atenção formal (pacientes) como à informal (cuidadores), além do custo total. Destaca-se que recentemente, após um painel entre especialistas, nova versão do questionário foi elaborada, o RUD versão 4.0 (WIMO ET AL., 2012).

\section{Resultados}

No que tange à revisão de literatura, e conforme já esperado, os estudos são oriundos de países europeus.

$\mathrm{Na}$ busca realizada nas bases citadas, foram localizados inicialmente 76 artigos, dos quais apenas sete contemplaram os critérios de inclusão e exclusão, sendo que os demais ou eram artigos repetidos ou não reportavam nenhum valor sobre custo ou versavam sobre tempo dispensado pelo cuidador ao paciente demenciado. Os resultados mais relevantes encontram-se na tabela 1. 
Tabela 1. Características dos estudos de avaliação econômica sobre síndrome demencial

\begin{tabular}{|c|c|c|c|c|c|c|c|}
\hline Autor/ano & $\begin{array}{r}\text { Perspectiva } \\
\text { do estudo }\end{array}$ & Tipo de estudo/método & amostra & $\begin{array}{r}\text { Grau de } \\
\text { severidade da } \\
\text { doença/ } \\
\text { outra variável }\end{array}$ & $\begin{array}{l}\text { Custo } \\
\text { direto }\end{array}$ & $\begin{array}{r}\text { Custo } \\
\text { indireto }\end{array}$ & Custo total \\
\hline $\begin{array}{l}\text { LINDHOLM, C. et } \\
\text { al., } 2012\end{array}$ & sociedade & prospectivo & 173 & $\begin{array}{r}\text { moderado e } \\
\text { severo }\end{array}$ & - & - & $\begin{array}{r}262000(\text { SEK } \cdot) / \text { moderado } \\
€ 30465,1 \text { ano } \\
519000(\text { SEK) / severo } \\
€ 60348,8 \text { ano }\end{array}$ \\
\hline $\begin{array}{l}\text { GUSTAVSSON, A. } \\
\text { et al., } 2010\end{array}$ & sociedade & observacional & 1385 & $\begin{array}{r}\text { leve/moderado/ } \\
\text { grave }\end{array}$ & $€ 1251,20$ & $€ 6568,80$ & $€ 7820$ (custo médio) ano \\
\hline $\begin{array}{l}\text { ÉRSEK, K et al., } \\
2010\end{array}$ & sociedade & $\begin{array}{r}\text { transversal/cost-of-illness } \\
(\mathrm{COI})\end{array}$ & 88 & $\begin{array}{r}\text { leve/moderado/ } \\
\text { grave }\end{array}$ & - & - & $\begin{array}{r}€ 6432 \text { (médio)- } \\
\text { domiciliado/ano } \\
€ 6086 \text { (médio)-asilado/ano }\end{array}$ \\
\hline $\begin{array}{l}\text { CODURAS, A. et } \\
\text { al., } 2010\end{array}$ & sociedade & $\begin{array}{r}\text { prospectivo (1 ano)/cost- } \\
\text { of-illness (COI) }\end{array}$ & 560 & $\begin{array}{r}\text { leve/moderado/ } \\
\text { grave }\end{array}$ & $\begin{array}{l}€ 366,73 \\
\text { (médio/ } \\
\text { mensal) }\end{array}$ & $\begin{array}{l}€ 1059 \\
\text { (médio/ } \\
\text { mensal) }\end{array}$ & $\begin{array}{r}€ 1425,73 € \text { (médio/ } \\
\text { mensal) }\end{array}$ \\
\hline $\begin{array}{l}\text { MESTERTON, J. } \\
\text { et al., } 2010\end{array}$ & sociedade & $\begin{array}{r}\text { transversal/ cost-of- } \\
\text { illness (COI) }\end{array}$ & 233 & $\begin{array}{r}\text { leve, moderado } \\
\text { e grave }\end{array}$ & - & - & $\begin{array}{r}\text { U\$S23.400 ano(leve) } \\
€ 17,475,72 \text { U\$S56.800 ano } \\
\text { (moderada) } € 42,419,72 \\
\text { U\$S71.400 ano (severa) } \\
€ 53,323,37\end{array}$ \\
\hline $\begin{array}{l}\text { JEDENIUS, E. et } \\
\text { al., } 2010\end{array}$ & serviço & $\begin{array}{r}\text { prospectivo/ cost-of- } \\
\text { illness (COI) }\end{array}$ & 30 & $\begin{array}{r}\text { atenção } \\
\text { primária/ } \\
\text { especialista } \\
\text { (diagnóstico } \\
\text { da doença) }\end{array}$ & - & - & $\begin{array}{r}\text { €477 APS (custo médio)/ } \\
\text { €1.115 especialista } \\
\text { (custo médio) }\end{array}$ \\
\hline $\begin{array}{l}\text { SøGAARD, R et } \\
\text { al., } 2009\end{array}$ & sociedade & $\begin{array}{r}\text { transversal/cost-of-illness } \\
(\mathrm{COI})\end{array}$ & 163 & leve & $€ 1070$ & $€ 950$ & $€ 2020$ mensal \\
\hline
\end{tabular}

Fonte: ECB: Euro foreign exchange reference rates

- 1 euro= 8,6 SEK (dezembro de 2012); 1euro = 1,339 USD (dezembro de 2010)

Em uma perspectiva comparativa, grosso modo, os estudos de Coduras et al. (2010), Søgaard et al. (2009) e Mesterton et al. (2010) apresentaram valores aproximados, caso se considere o custo em período anual. Por outro lado, os estudos de Gustavsson et al. (2010) e Érsek et al. (2010), também com valores aproximados entre si, encontraram valores menores do que os obtidos pelo primeiro grupo de estudos.

Diante de as pesquisas revelarem outras informações pertinentes, que ampliam o conhecimento sobre a relação entre a carga da doença e seu impacto em termos financeiros, e que em larga medida subsidia o conhecimento das necessidades sociais de saúde, um breve resumo sobre essas informações encontra-se abaixo.
Estudo publicado recentemente, realizado por Lindholm et al. (2012), na Suécia, revela que o custo para a sociedade (custo formal e informal) da síndrome demencial varia de 262000 SEK por ano, para o caso de portadores no grau moderado, a 519000 SEK por ano, para os pacientes com o grau severo da doença. O estudo identificou, por meio de análise univariada, que tanto as AVD (Atividades de Vida Diária) quanto as AIVD (Atividades Instrumentais de Vida Diária) estão relacionadas aos custos. Por meio de análise multivariada, também demonstrou que o nível de cognição não é estatisticamente significante no que tange ao total dos custos. Já a análise que comparou a dependência ou a não dependência de qualquer uma das funções básicas das AVD 
mostra alteração significativa dos custos. A conclusão do estudo é de que o nível da funcionalidade deteriorada, a qual requer um importante suporte social, prediz o aumento dos custos da atenção ao portador de síndrome demencial (LINDHOLM ET AL., 2012). Mesterton et al. (2010), em alguma medida, obtiveram resultados semelhantes no que se refere às funções das AVD e o impacto no custo da atenção, ressaltando inclusive o peso nesse custo do tipo de acomodação (moradia), que tem uma dada cobertura, nos países do norte europeu, por meio da proteção social.

Em estudo multicêntrico (12 países europeus) do tipo observacional, Gustavsson et al. (2010) estimaram o custo médio anual em 7820 euros (95\% CI: 7194- 8446), sendo que $54 \%$ desse valor estão atrelados ao custo informal, $16 \%$ ao custo médico direto e $30 \%$ relacionado aos serviços comunitários. Conclusão relevante do estudo alude que há substancial diferença em relação ao uso de recursos entre os tipos de custo (formal e informal) entre os países do norte, os do sul e da parte ocidental da Europa. O custo formal é baixo e o informal é alto no sul da Europa, se comparado com o norte e a parte ocidental. Assim como as AIVD estão fortemente correlacionadas com o custo informal e o índice de Katz (avalia o autocuidado) com os custos formais (GUSTAVSSON ET AL., 2010).

Em outra vertente, estudo de corte transversal utilizou o método cost-of-illness (COI) para avaliar o custo dos pacientes domiciliados e o dos pacientes asilados, que advém de reembolso oficial, para determinar a carga da doença do ponto de vista da sociedade. Foram incluídos na sua amostra pacientes e cuidadores de três GP (general practitioner) na Hungria. Inferiu-se um custo médio anual de 6432 euros para o paciente domiciliado e 6086 euros para o paciente asilado. O estudo efetivou a previsão do impacto financeiro para a população total do País em aproximadamente 846,8 milhões de euros, sendo que $55 \%$ em custos diretos, $9 \%$ em custos indiretos (não médico) e 36\% em custo informal (ÉRSEK ET AL., 2010). Os custos na Hungria em relação aos pacientes demenciados são mais baixos se comparados com os dos demais países da Europa ocidental (CODURAS ET AL., 2010).

$\mathrm{Na}$ Espanha, estudo prospectivo com duração de um ano, em pacientes nos três graus de severidade da patologia, evidenciou um custo mensal médio de 1316,22 euros no início da coleta de dados e de 1425,73 euros no $12^{\circ}$ mês, ou seja, um acréscimo de $10,08 \%$ ao longo do período. Já no que se refere ao custo não médico, que representa 74,30 \%, o valor encontrado foi de 1059 euros, o qual decresceu no período estudado 4,30 euro/mês. No que concerne à atenção, que apresentou um custo médio de 366,66 euros, o referido custo cresceu 136,94 euros no período, impactado tanto pelo custo da medicação como pela utilização de serviços de enfermagem domiciliar e institucionalização. Vale ressaltar que $87 \%$ do custo total na Espanha não é financiado pelo Serviço Nacional de Saúde e a maior parte desse custo está relacionado com o custo informal (70,86\%); ou seja, quase $88 \%$ do custo da doença é financiado pela família (CODURAS ET AL., 2010).

Poucos estudos avaliaram o custo da doença associado ao custo com o deslocamento (transporte) relativo à busca por cuidados de saúde e outras atividades relacionadas à síndrome e o tempo perdido de pacientes no grau leve da doença. Søgaard et al. (2009), ao ponderarem a relação custo público e privado, a estimaram em 1:1. Os autores chamam a atenção para o fato de o dispêndio privado incorrer em possível desequilíbrio para o planejamento do sistema público no que tange à relação oferta-demanda por bens e serviços de saúde, levando a um prejuízo da equidade da atenção à saúde.

Em uma perspectiva diferenciada de avaliação do impacto financeiro da doença, Jedenius et al. (2010) analisaram o custo do diagnóstico da doença quando efetivada na atenção primária e quando realizada por um especialista. Os autores concluíram que 
o diagnóstico é dado entre duas semanas a dois meses e que os custos são diferenciados entre as duas modalidades de atenção, sendo os do especialista mais altos do que os da atenção primária.

\section{Discussão}

Os custos da síndrome se devem a dois fatores: de um lado, a demanda crescente por cuidados e a continuidade da atenção; por outro, a necessidade da presença do cuidador que, com frequência, precisa abandonar seu trabalho para cuidar do enfermo, o que gera perda de renda, justamente quando aumentam os gastos.

Diversos autores afirmam que o crescente aumento do custo da atenção social e da saúde do idoso portador de síndrome demencial não encontra, no numerário público, a capacidade correspondente de atender à totalidade das necessidades (WIMO; PRINCE, 2010). Não obstante, existe certa reticência em planejar o futuro dessa assistência, uma vez que não desperta o interesse da classe política e o corpo técnico não tem oferecido soluções atraentes. Ou seja, o grande desafio para os governos é desenvolver e melhorar os serviços com foco no diagnóstico precoce, no fornecimento de apoio para a comunidade, bem como respostas tanto do setor saúde quanto da assistência social. A integração dos processos da saúde e os de cunho social é necessária para garantir as especificidades desse grupo da população.

Os resultados dos estudos advindos da revisão demonstram que a abrangência das informações pertinentes e relacionadas não só às condições de saúde como também de cunho social respalda uma avaliação de impacto financeiro da patologia, assim como também revela o panorama do comportamento dos países no que concerne à proteção social aos idosos demenciados.

Embora existam na literatura outros questionários sobre utilização de recursos em relação à demência, a escolha do questionário $R U D$ como direcionador para o estudo em questão se ateve não somente ao fato de já ter sido utilizado em várias pesquisas em diferentes países, mas também por ter uma versão em português (Portugal) e possibilitar em alguma medida um balizamento entre resultados de diferentes estudos. É importante citar que a variável custo apresenta em termos estatísticos um comportamento assimétrico, em que a distribuição gama é não normal e o custo total corrigido por uma lognormal. No caso da variável se referir ao gasto per capita, o recomendado é a utilização do Purchasing Power Parity (PPP), cuja técnica utiliza o valor relativo das moedas, ao estimar o montante de ajustamento necessário entre os países, no sentido de que a troca seja equivalente ao poder de compra de cada moeda.

As diferenças encontradas nos valores dos sete estudos são decorrentes não apenas do fato de eles terem sido realizados em momentos distintos em termos temporais, mas também pelo incremento na utilização e a decorrente valoração monetária dos recursos utilizados pelos pacientes ou por seus cuidadores. Também vale citar que nos países desenvolvidos a utilização de recursos em relação aos itens afetos às políticas sociais é mais expressiva e que, portanto, gera um custo maior, o que dificulta sobremodo a comparabilidade de resultados.

Entre os diferentes modelos/métodos para estudos nesta área do conhecimento, o custo-da-doença (COI), considerado o primeiro método descritivo de avaliação econômica no campo da saúde, tem sido o método de escolha para a avaliação de impacto financeiro para a sociedade, tanto na vertente topdown quanto, e principalmente, na vertente bottom-up, que apresenta como vantagem a admissão de estratificação da severidade das doenças e a verificação da variabilidade ao se apropriar/compor os diferentes tipos de custo (TARRICONE, 2006; WIMO; JONSSON; GUSTAVSSON, 2009). O método tem também a propriedade 
de mensurar a perda de produtividade e é pautado pela constatação contrafactual de que o seu benefício advém do conhecimento do custo poupado ao se prevenir ou minimizar os efeitos de uma dada patologia (SEGEL, 2006). Ademais, o método é construído a partir das perspectivas epidemiológica (prevalência ou incidência) e econômica (grosscosting ou micro-costing).

Utilizando o método COI, foi elaborado na Inglaterra estudo que dimensiona com propriedade o impacto financeiro, com valores relativos ao ano de 2008 , ao assumir um custo de $\mathfrak{E} 23$ bilhões/ano (aproximadamente $€ 21$ bilhões referentes ao câmbio de 2008), entre custos diretos e perda de produtividade (custo indireto), para um conjunto de 820 mil pacientes demenciados. Nesse estudo, o custo da atenção social é de $£ 9$ bilhões ( $€ 8$ bilhões) $-40 \%$ do total -, o dos cuidados não remunerados na ordem de $\mathfrak{E}$ 12,4 bilhões ( $€$ 11,4 bilhões) $-55 \%$ - e o da atenção médica de $£ 1,2$ bilhões ( $€ 1,1$ bilhão) $-5 \%$ - além de um custo adicional de $£ 29$ milhões ( $€ 26,7$ milhões) decorrente da perda da produtividade. É também ressaltado pelo estudo que o custo com a síndrome demencial é duas vezes maior do que com câncer e três vezes maior do que com doenças do coração (LUENGO-FERNANDEZ; LEAL; GRAY, 2010).

O único estudo realizado no Brasil revela que os gastos das famílias com pacientes portadores de síndrome demencial correspondem, em média, a $66 \%$ da receita familiar. No caso do paciente em estágio inicial, este percentual chega a $75 \%$, possivelmente explicado pela necessidade de investimentos (readequações e reformas) no domicílio; no estágio avançado atinge 58\% e, na presença de comorbidade, $80 \%$ (VERAS ET AL., 2007). Deve-se ressalvar que o estudo não incorporou na análise o gasto com $o$ cuidador formal. É importante mencionar que o estudo, ao tratar dos gastos/despesas e não dos custos da atenção ao portador e do cuidador na síndrome demencial, não desvela o seu impacto financeiro, bem como não oferece subsídios em relação às reais necessidades sociais e de saúde no que tange ao seu enfrentamento.

\section{Conclusão}

A importância desta revisão é chamar a atenção daqueles que pesquisam na área do envelhecimento, bem como suscitar a necessidade da elaboração de estudos deste porte, não apenas para subsidiar os tomadores de decisão no âmbito político, mas também para sensibilizar aqueles que detêm o poder discricionário no que concerne ao apoio financeiro para a elaboração de pesquisas.

No Brasil, de maneira inconteste, apenas um estudo foi elaborado sobre os gastos das famílias, e ainda não se logrou a implementação de uma pesquisa sobre o real impacto financeiro da síndrome demencial.

Certamente esse fato causa espécie. Isto porque há diversos grupos de pesquisa que desenvolvem pesquisas na área de conhecimento do envelhecimento. Existem, entretanto, possíveis explicações para essa constatação: ou a não obtenção de recursos financeiros das agências financiadoras para elaborar estudos dessa ordem, por não ser prioridade em termos de investigação acadêmica, ou a patologia ainda não está elencada como um problema de saúde pública.

De qualquer forma, e na perspectiva de que esse importante problema mundial de saúde pública ainda se torne relevante no contexto brasileiro, dispôs-se um conjunto de informações que, em grande medida, pode subsidiar o dimensionamento provável do custo e, por conseguinte, o impacto da síndrome demencial tanto no que se refere ao setor público quanto na perspectiva da sociedade, aí incluídos aquele desembolsado diretamente pelas famílias (out-of-pocket). 


\section{Referências}

\begin{abstract}
ALMEIDA, F. N.; SANTANA, V. S.; PINHO, R. Estudo epidemiológico dos transtornos mentais em uma população de idosos: área urbana de Salvador. Jornal Brasileiro de Psiquiatria, Rio de Janeiro, v. 33, n. 2, p. 114-120, 1984.
\end{abstract}

BLAY, S. L.; MARI, J. J.; RAMOS, L. R. O uso do "face-hand test" como instrumento para rastrear as síndromes psicorgânicas: estudo piloto. Revista Saúde Pública, São Paulo, v. 23, n. 5, p. 395-400, 1989.

BRASIL. Ministério da Saúde. Portaria n ${ }^{\circ}$ 399/GM, de 22 de fevereiro de 2006. Divulga o Pacto pela Saúde 2006 - Consolidação do SUS e aprova as Diretrizes Operacionais do Referido Pacto. Diário oficial [da] União. Brasília, DF, 22 fev. 2006. Disponível em: <http://dtr2001.saude.gov.br/sas/PORTARIAS/ Port2006/GM/GM-399.htm>. Acesso em: 20 mar. 2014.

Política Nacional de Saúde do Idoso. Sem descrição. Diário Oficial [da] União, Ministério da Saúde, Brasília, DF, 13 de dezembro de 1999, nº 237-E, Seção 1. p. 20-24.

Ministério da Saúde. Portaria GM/MS n ${ }^{\circ} 703$, de 12 de abril de 2002. Dispõe sobre a instituição do Programa de Assistência aos Portadores da Doença de Alzheimer no âmbito do Sistema Único de Saúde e dá outras providências. Diário Oficial [da] União, Brasília, DF, 12 abr. 2002.

Ministério da Saúde. Portaria SAS/MS no 843, de 6 de novembro de 2002. Aprova o Protocolo Clínico e Diretrizes Terapêuticas - Demência por Doença de Alzheimer - Rivastigmina, Galantamina e Donepezil e dá outras providências. Diário Oficial [da] União, Brasília, DF, 6 nov. 2002.

CHAPPELL, N. L. The future of health care in Canada. Journal Social Policy, Cambridge, v. 22, n. 4, p. 487-505, out. 1993.

CODURAS, A. et al. Prospective one-year cost-of-illness study in a cohort of patients with dementia of Alzheimer's disease type in Spain: the ECO study. American Journal of Alzheimer Disease, Weston, v. 19, n. 2, p. 601-15, 2010.

ÉRSEK, K. et al. Costs of Dementia in Hungary. Journal of Nutrition, Health \&t Aging, Paris, v. 14, n. 8, p. 633-39, 2010.
GALLUCI, N. J.; TAMELINI, M. G.; FORLENZA, O. V. Diagnóstico diferencial das demências. Revista Psiquiatria Clínica, São Paulo, v. 532, n. 3, p. 119-130, 2005.

GUSTAVSSON, A. et al. ICTUS Study Group. Differences in resource use and costs of dementia care between European countries: baseline data from the ICTUS study. The Journal of Nutrition, Health \& Aging, Paris, v. 14, n. 8, p. 648-54, out. 2010.

HERRERA, E. J. R. et al. Epidemiologic survey of dementia in a community-dwelling Brazilian population. Alzheimer Disease Associated Disorders, Hagerstown, v.16, n.2, p.103-8, abr./jun. 2002. Disponível em: <http://www.dementia2010.org/reports/ DEMENTIA2010FULL.pdf >. Acesso em: 17 maio 2011.

JEDENIUS, E. et al. The cost of diagnosing dementia in a community setting. International Journal of Geriatric Psychiatry, Chichester, v. 25, n. 5, p. 476-82, maio 2010.

JÖNSSON, L.; WIMO, A. The cost of dementia in Europe: a review of the evidence, and methodological considerations. Pharmacoeconomics, Auckland, v. 27, n. 5, p. 391-403, 2009.

LINDHOLM, C. et al. Costs explained by function rather than diagnosis-results from the SNAC Nordanstig elderly cohort in Sweden. International Journal of Geriatric Psychiatry, Manchester, v. 28, n. 4, p. 454-62, maio 2013.

LUENGO-FERNANDEZ, R.; LEAL, J.; GRAY, A. Dementia 10. The economic burden of dementia and associated research funding in the United Kingdom. Health Economics Research Centre, University of Oxford for the Alzheimer's Research Trust. Disponível em: <http://www.herc.ox.ac.uk/pubs/downloads/dementiafullreport>. Acesso em: 20 maio 2012.

MESTERTON, J. et al. Cross sectional observational study on the societal costs of Alzheimer's disease. Current Alzheimer Research, Saif Zone, v. 7, n. 4, p. 35867, jun. 2010.

MORAGAS, R. M. El Centro de día para enfermos de Alzheimer. Barcelona: UCB Pharma, 1994.

NITRINI, R. et al. Incidence of Dementia in a Community-Dwelling Brazilian Population. Incidence 
of Dementia in a Community-Dwelling Brazilian Population. Alzheimer Disease \& Associated Disorders, Hagerstown, v. 18, n. 4, p. 241-246, 2004.

\section{SEGEL, J. E. Cost-of-Illness Studies - A Primer. RTI-} UNC Center of Excellence in Health Promotion Economics, 2006. Disponível em: <http://www.rti.org/ pubs/coi_primer.pdf >. Acesso em: 17 maio 2011.

SØGAARD, R. et al. Private costs almost equal health care costs when intervening in mild Alzheimer's: a cohort study alongside the DAISY trial. Health Services Research, v. 25, n. 9, p. 215, nov. 2009. Disponível em: $<$ http://www.biomedcentral.com/1472-6963/9/215>. Acesso em: 20 jun. 2012.

TARRICONE, R. Cost-of-illness analysis. What room in health economics? Health Policy, Limerick, v. 77, n. 1 , p. $51-63,2006$

VERAS, R. P. et al. Avaliação dos gastos com o cuidado do idoso com demência. Revista Psiquiatria Clínica, São Paulo, v. 34, n. 1, p. 5-12, 2007.

VERAS, R. P.; COUTINHO, E. S. F. Estudos de Prevalência de depressão e síndrome cerebral orgânica na população de idosos. Revista de Saúde Pública, São Paulo, v. 25, n. 4, p. 209-17, 1991.

WIMO, A. et al. Application of Resource Utilization in Dementia (RUD) instrument in a global setting. Alzheimer's \& Dementia, Orlando, v. 9, n. 4, p. 429-35, jul. 2013.

WIMO, A. et al. Evaluation of the healthcare resource utilization and caregiver time in anti-dementia drug trials. In: WIMO, A. (Org.). Health Economics of Dementia. Chichester: Wiley, 1998. p. 465-477.
WIMO, A. et al. The economic impact of dementia in Europe in 2008-cost estimates from the Eurocode project. International Journal of Geriatric Psychiatry, Chichester, v. 26, n. 8, p. 825-32, ago. 2011

WIMO, A.; JÖNSSON, L.; GUSTAVSSON, A. Cost of illness and burden of dementia - The base option. Alzheimer Europe, 2009. Disponível em: <http:// www.alzheimer-europe.org/FR/Research/EuropeanCollaboration-on-Dementia/Cost-of-dementia/Costof-illness-and-burden-of-dementia >. Acesso em: 17 maio 2011.

WIMO, A.; JONSSON, L.; WINBLAD, B. An estimate of the worldwide prevalence and direct costs of dementia in 2003. Dementia Geriatric Cognitive Disorders, Basel, v. 21, n. 3, p. 175-81, 2006.

WIMO, A.; PRINCE, M. Alzheimer's Disease International World Alzheimer Report 2010. The Global Economic Impact of Dementia. Alzheimer's Disease International September 2010. Disponível em: <http://www.alz.co.uk/research/files/ WorldAlzheimerReport2010.pdf $>$. Acesso em: 20 maio 2011.

Recebido para publicação em dezembro de 2014

Versão final em janeiro de 2015

Conflito de interesse: inexistente

Suporte financeiro: não houve 